\title{
Design of Low Voltage and High-Speed BiCMOS Buffer for Driving Large Load Capacitor
}

\author{
Maede Kaviani $^{\mathrm{a}}$, Hojjat Sharifi ${ }^{\mathrm{b}, *}$, Mahdi Dolatshahi $^{\mathrm{a}}$ and Keivan $\mathrm{Navi}^{\mathrm{c}}$ \\ ${ }^{a}$ Department of Computer Engineering, Najafabad Branch, Islamic Azad University, Najafabad, Esfahan \\ PO BOX: 8514143131, Iran \\ ${ }^{b}$ Department of Computer Engineering, Vali-e-Asr University of Rafsanjan, Rafsanjan PO BOX: 518, Iran \\ ${ }^{c}$ Department of Computer Science and Engineering, Shahid Beheshti University, GC, Tehran PO BOX: \\ 1983963113, Iran
}

\begin{abstract}
BICMOS circuits are interesting for designers when a high speed output driver is required especially in I/O circuits. Buffer is an important block in high speed circuits, so designing a buffer with high drive capability has a great effect on circuits with large load capacitor. This paper presents a new BiCMOS buffer which uses $32 \mathrm{~nm}$ technology node for CMOS transistors and 0.18um technology node for BJT transistors. The proposed buffer operates properly in voltage ranges from $0.8 \mathrm{v}$ to $1.5 \mathrm{v}$. The capacitor range is from $0.5 \mathrm{pf}$ to $200 \mathrm{pf}$; the overshoot of the output in this capacitor range is less than $10 \%$ of the supply voltage that is negligible. The proposed design has improvements in delay for about \%88 respectively compared to similar CMOS buffers with high capacitor values.
\end{abstract}

Index Terms: Buffer, BiCMOS, High load, Capacitor, Low power, Inverter.

(C) 2016 Published by MECS Publisher. Selection and/or peer review under responsibility of the Research Association of Modern Education and Computer Science.

\section{Introduction}

In recent years, the advantages of CMOS over BiCMOS technology such as speed, power, and cost have been proven. Hence, many researches focus on investigating the aspects of low voltage, low power, and high speed for this dominant semiconductor technology. Using lower power supply voltage is one of the major solutions for decreasing device power consumption. Nowadays, circuits are designed to operate within the domains near the threshold-voltage as a result of these efforts to reduce supply voltage $\left(\mathrm{V}_{\mathrm{th}}\right)$ [1] [2].

Circuits with high current capability which use Bipolar and CMOS technologies combination, named BiCMOS. BiCMOS technology is beneficial especially when a high speed output driver is required. The circuit

* Corresponding author. Tel.: 982129904109

E-mail address: h.sharifi@vru.ac.ir 
designers' ultimate goals are to scale down supply voltage, and decrease the propagation delay along with power consumption concurrently. Since these parameters are not achievable at the same time, so making a tradeoff among them should be considered [3] [4]. It is worth noting that high capacitive loads are often appeared in I/O circuits [10][11]. Buffers are crucial blocks in high speed circuits; therefore, a buffer with high drive capability would be significantly effective for circuits with large load capacitor [2] [5] [7] [8]. In this paper a BiCMOS buffer is proposed with a supply voltage of $0.8 \mathrm{v} \sim 1.5 \mathrm{v}$.

Due to the fact that the recently suggested designs for CML buffers in enjoy a great deal of advantages, since these buffers have high static power consumption, suggesting a circuit with a low rate of static power consumption can be of great interest. To improve the proposed design it can be also changed into tapered buffer in order to not only reduce the power consumption, but also decrease the delay of the circuit. The proposed scheme in this paper, can be used to design the current mode BiCMOS circuit which can be applied in ripple adder circuits. [5] [8]

The rest of the paper is organized as follows: the former investigations about BiCMOS inverter gate designs are demonstrated in Section 2. Section 3 proposes a new BiCMOS design which will be optimized. The simulation results and comparisons are presented in section 4. Finally, Section 5 comes with the conclusion.

\section{Related Work}

Over the past decades, different BiCMOS circuits were presented. Power, delay, power delay product (PDP), high swing capability and overshoot are among major parameters for evaluating BiCMOS circuits. Researches on designing of BiCMOS circuits mainly focus on high swing designs besides low power circuits. For instance, high swing circuits using resistance elements are not desirable due to their high power consumption. In BiCMOS circuits, overshoot values under $10 \%$ of supply voltage are negligible.

Some BiCMOS inverters have been studied in [4], [6], [8] and [9]. In this paper two example of BiCMOS inverter circuits are discussed and compared to each other as conventional designs which are shown in Fig. 1 (a) and (b). The circuit operation of Fig. 1 (a) is described as following. If the input voltage is ' 0 ', the transistor P1 is $\mathrm{ON}$, where $\mathrm{N} 1$ and $\mathrm{N} 2$ are $\mathrm{OFF}$. Therefore $\mathrm{V}_{\mathrm{DD}}$ is transferred to $\mathrm{B} 1$ node through $\mathrm{P} 1$. Logic ' 1 ' on $\mathrm{B} 1$ turns the Q1 ON and causes $\mathrm{V}_{\text {out }}$ to be '1'. High voltage on B1 node turns the $\mathrm{N} 3$ transistor ON, then Q2 is being $\mathrm{OFF}$. When the input voltage equals to ' $\mathrm{V}_{\mathrm{DD}}$ ', $\mathrm{P} 1$ is $\mathrm{OFF}$ and $\mathrm{N} 1$ and $\mathrm{N} 2$ are $\mathrm{ON}$. Consequently $\mathrm{V}_{\mathrm{GND}}$ is passed on $\mathrm{B} 1$ node through $\mathrm{N} 1$ transistor. As $\mathrm{V}_{\mathrm{B} 1}=$ ' 0 ', so Q1 and $\mathrm{N} 3$ are turned OFF. Consequently transistor Q2 is ON, C1 Capacitance is discharged and causes $V_{\text {out }}$ to be logic ' 1 ' [4] [6] [8].

Fig.1 (b) shows another BiCMOS inverter design. The circuit works as below. When input voltage equals to ' 0 ', transistor $\mathrm{P} 1$ is $\mathrm{ON}$ and transistor $\mathrm{N} 1$ is OFF. So transistors $\mathrm{Q}_{1}$ and $\mathrm{N} 2$ are $\mathrm{ON}$ and output voltage will be logic ' 1 '. When input voltage is equal to ' 1 ', $\mathrm{N} 1$ is $\mathrm{ON}$. So $\mathrm{Q}_{1}$ is being OFF and P2 and Q2 are ON. Hence, $\mathrm{C} 1$ capacitance is discharged and consequently output voltage is equal to ' 0 ' [9].

The functionality of these circuits requires that one of the BJTs to be OFF to avoid an unknown output caused by competing two BJTs to concurrently pull down and up the output. These circuits which have been used as inverter gates have the following specifications: the minimum supply voltage is $3.3 \mathrm{v}$. In addition when the output logic is ' 1 ' the output voltage will be decreased by the amount of BJT threshold voltage.

Other proposed designs in recent years, which is mentioned in the introduction, is CML buffers. Although they have acceptable capabilities, but according to static power consumption, they are not in desirable condition. Therefore, there are many efforts have been made to reduce the static power by designing the improved schemes. Generally, in order to increasing the speed of buffers, the sequence buffers are used, which are called tapered buffers, and they are in the cascaded buffers category. This method is exploited for CML buffers and conventional CMOS buffers. [5] [10] [12]. 


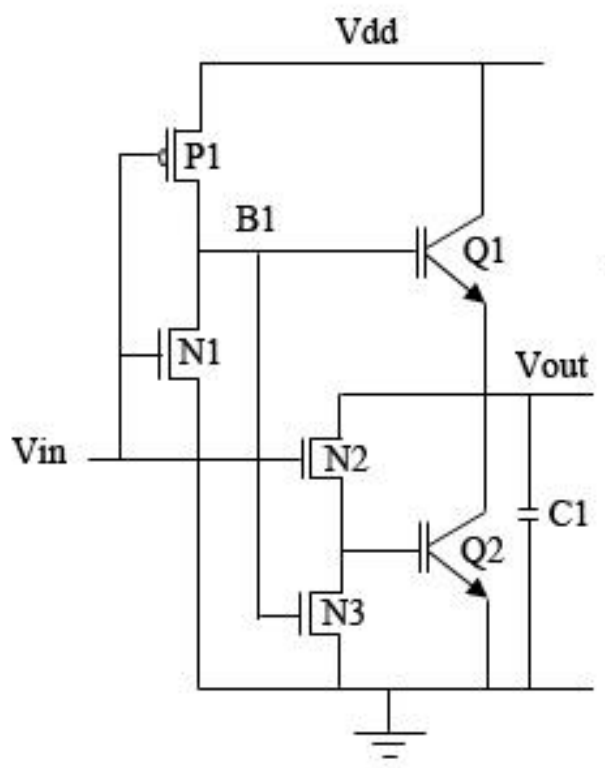

(a)

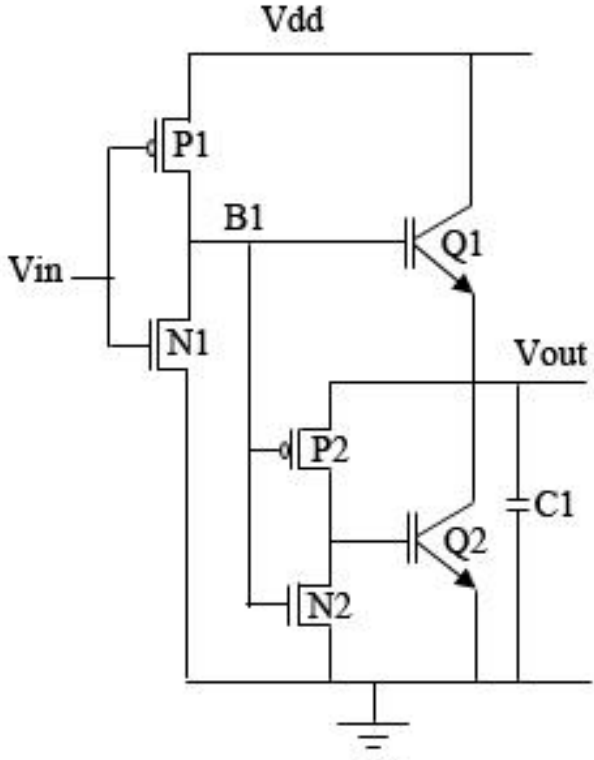

(b)

Fig.1. (a) A Conventional inverter Circuit. (b) An inverter which Uses PMOS Transistor.

\section{HDBiCMOS (High Drive BiCMOS) buffer}

The HDBiCMOS circuit, as a kind of BiCMOS buffer, uses cascaded CMOS and bipolar inverters. Fig. 2 illustrates the proposed BiCMOS buffer that its operation is described as follows: if $\mathrm{V}_{\mathrm{IN}}=0, \mathrm{P} 1$ and $\mathrm{Q} 2$ transistors will be $\mathrm{ON}$, so capacitor $\mathrm{C} 1$ will be charged, and capacitor $\mathrm{C} 2$ will be discharged and voltage output would be equal to ' 0 '. When the input voltage is $\mathrm{V}_{\mathrm{DD}}, \mathrm{N} 1$ is $\mathrm{ON}$ and $\mathrm{C} 1$ will be discharged. So Q1 is ON and the output logic will be ' 1 '.

The problem of such a design is the voltage level variation of node $\mathrm{C}$. when $\mathrm{C}$ is in high voltage level, so Q2 is $\mathrm{ON}$ and the voltage level of $\mathrm{C}$ has been decreased gradually and this causes that $\mathrm{Q} 1$ will be turning ON. Consequently this problem make the output voltage will be unknown. This problem also happened similarly when the voltage level of $\mathrm{C}$ is low.

The only solution for this problem is to prevent Q1 and Q2 to be simultaneously in active region. To do so, we suggest reducing the supply voltage. By applying lower supply voltages, when the voltage level of $\mathrm{C}$ is high, $\mathrm{Q} 2$ is $\mathrm{ON}$ and by decreasing the voltage level of $\mathrm{C}$, Q1 is still OFF since $\mathrm{V}_{\mathrm{EB}}(\mathrm{Q} 1)<\mathrm{V}_{\mathrm{th}}(\mathrm{Q} 1)$. When the voltage level of $\mathrm{C}$ is low, Q1 is ON and Q2 is OFF and will be remained OFF since $\mathrm{V}_{\mathrm{BE}}(\mathrm{Q} 2)<\mathrm{V}_{\mathrm{th}}(\mathrm{Q} 2)$.

Cascading two CMOS inverters results in a CMOS buffer (Fig. 3). These circuits are compared according to previously mentioned parameters. Compared features include: power, propagation delay, power delay product (PDP) and the correctness of circuit functionality. As long as load capacitor value is small, both the BiCMOS and CMOS buffers have similar performances. By raising the lead capacitor values the output delay will be increased. Where, the CMOS buffer cannot work properly, the BiCMOS buffer have a perfect functionality. 


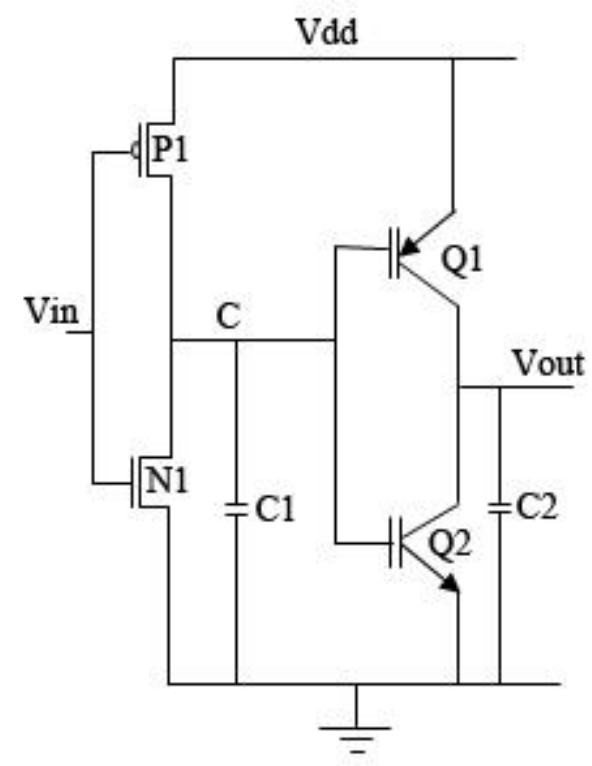

Fig.2. The Proposed Bicmos Buffer (HDBiCMOS)

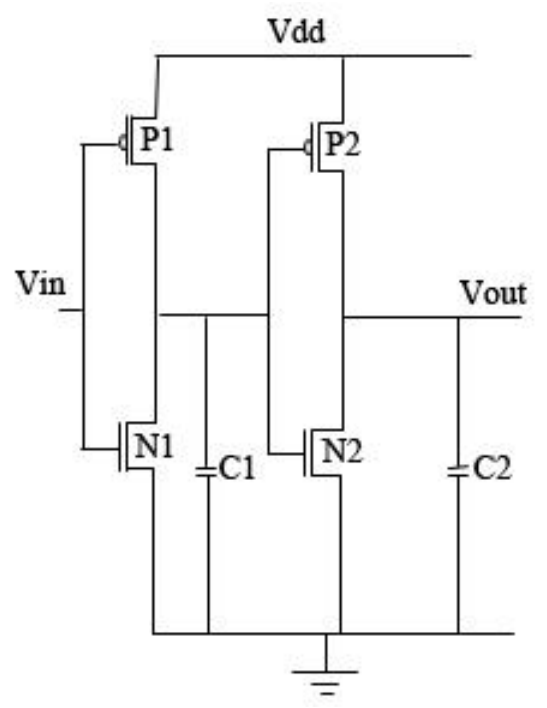

Fig.3. CMOS Buffer Circuit

As explained above, the circuits in Fig. 1 (a) and (b) operate with at least $3.3 \mathrm{v}$ supply voltages. In the proposed buffer reducing the voltage would solve the problem at $\mathrm{C}$ node; so the output of circuit would be acceptable. Regarding the supply voltage of modern circuits, against classic BiCMOS circuits, the proposed buffer operates perfectly in modern ICs. In order to reduce the buffer delay in large load capacitor, the proposed buffer was modified. In the modified buffer by adding an inverter we can charge and discharge the capacitor $\mathrm{C} 1$ faster. So we can achieve less delay. 


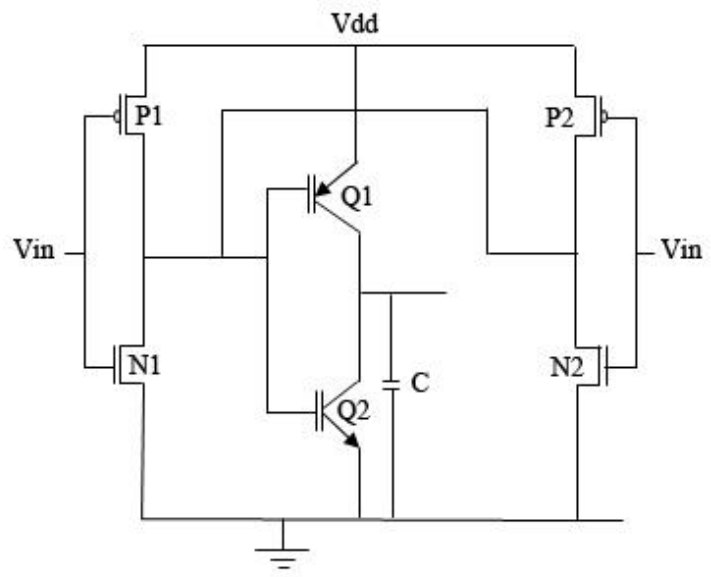

Fig.4. Proposed Modified HDBiCMOS Buffer

\section{Simulation Result}

The proposed HDBiCMOS circuit and the other previous circuits are simulated and compared. It is worth mentioning that TSMC 0.18 um technology for BJT transistors and 32nm technology for CMOS transistors are employed. Fig. 5 illustrates the transient respond of CMOS versus HDBiCMOS buffer outputs. The simulations are performed in the $1 \mathrm{~V}$ supply voltage and different load capacitors. HDBiCMOS circuits have significantly better functionality and less delay than CMOS circuits. HDBiCMOS circuit has bipolar transistors that provide bigger current that increase driving capability and faster charging and discharging for capacitors. According to the simulation results, by increasing the capacitor value more than 60pf, the CMOS buffer delay will be raised. Moreover, for large load capacitors, the CMOS buffer could not fully charges the capacitor, consequently resulting in output failure. A comparison of power, delay, and PDP values between HDBiCMOS and CMOS buffers is demonstrated in table 1. The capacitor values are 30pf, 60pf, 65pf, and 100pf. The output of CMOS buffer circuit using $1 \mathrm{~V}$ supply voltage with capacitor values more than $60 \mathrm{pf}$ will be failed.

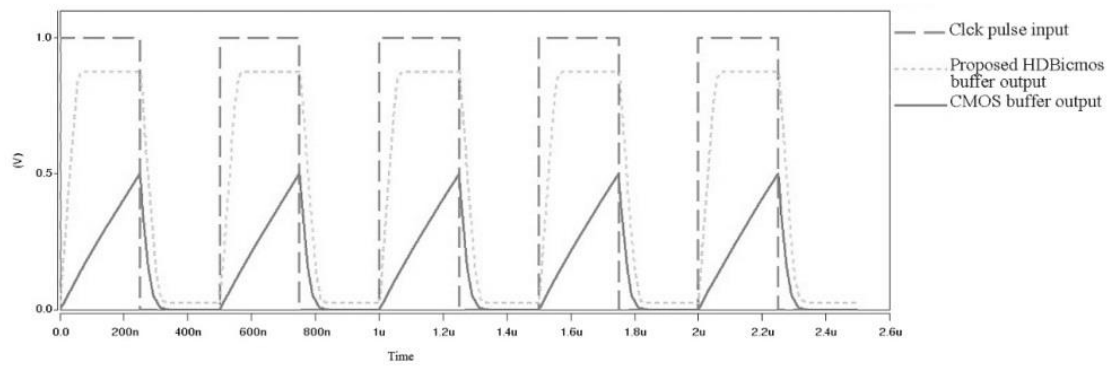

Fig.5. Output Comparison between HDBiCMOS Proposed Buffer and CMOS Buffer.

The comparison of delay and PDP values of both CMOS and proposed BiCMOS buffers in different supply voltages are shown in Fig. 6. As it is illustrated in the Fig. 6 by decreasing the supply voltage, PDP of the proposed buffer circuit is better than CMOS buffer, but delay of proposed buffer is entirely better than CMOS buffer. 
Delay, power consumption and PDP of the proposed HDBiCMOS buffer are compared to the modified proposed HDBiCMOS buffer. Based on the simulation results, delay at capacitor values of 10pf to 200pf are evaluated, which shows $38 \%$ improvement at least compared to the proposed HDBiCMOS buffer circuit (Table 2). To improve proposed circuit instead of using transistors with $\mathrm{W} / \mathrm{L}$ ratio size on the first stage of the circuit, we can use transistors with $\mathrm{W} / 2 \mathrm{~L}$ ratio size on two stages as it is illustrated in Fig. 4 . The main reason for this improvement is prevents the formation of large gate capacitor.

Table 1. Comparison of BiCMOS and CMOS in terms of Power, Delay, and PDP

\begin{tabular}{|c|c|c|c|c|}
\hline $\begin{array}{l}\text { Capacitor } \\
\text { Value }\end{array}$ & Circuit & Delay $(* e-8)$ & PDP(*e-11) & Power(*e-4) \\
\hline \multirow{2}{*}{$\begin{array}{l}\text { 100pf Load } \\
\text { Capacitor } \\
\text { Value }\end{array}$} & $\begin{array}{c}\text { proposed } \\
\text { HDBiCMOS buffer }\end{array}$ & 8.11 & 2.11 & 2.60 \\
\hline & CMOS buffer & \multicolumn{3}{|c|}{ Failure } \\
\hline \multirow{2}{*}{$\begin{array}{l}\text { 65pf Load } \\
\text { Capacitor } \\
\text { Value } \\
\end{array}$} & $\begin{array}{c}\text { proposed } \\
\text { HDBiCMOS buffer }\end{array}$ & 3.32 & 1.48 & 4.47 \\
\hline & CMOS buffer & \multicolumn{3}{|c|}{ Failure } \\
\hline \multirow{2}{*}{$\begin{array}{l}\text { 60pf Load } \\
\text { Capacitor } \\
\text { Value }\end{array}$} & $\begin{array}{c}\text { proposed } \\
\text { HDBiCMOS buffer }\end{array}$ & 4.90 & 1.14 & 2.33 \\
\hline & CMOS buffer & 25.2 & 1.21 & 0.476 \\
\hline \multirow{2}{*}{$\begin{array}{l}\text { 30pf Load } \\
\text { Capacitor } \\
\text { Value }\end{array}$} & $\begin{array}{c}\text { proposed } \\
\text { HDBiCMOS buffer }\end{array}$ & 1.56 & 0.614 & 3.92 \\
\hline & CMOS buffer & 12.6 & 0.650 & 0.517 \\
\hline
\end{tabular}

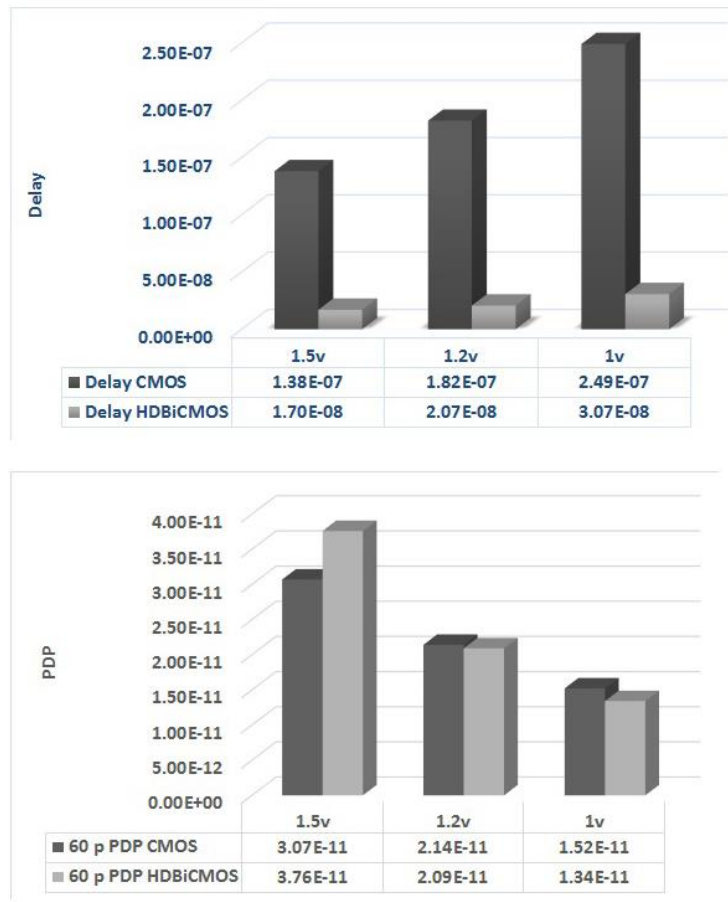

Fig.6. The Delay and PDP Comparison between HDBiCMOS and CMOS Buffers. 
Table 2. Consumption of CMOS Buffer and Proposed HDBiCMOS in terms of Propagation Delay, Power Consumption and PDP.

\begin{tabular}{|c|c|c|c|c|c|c|}
\hline \multirow{2}{*}{ CL(pf) } & \multicolumn{3}{|c|}{ Proposed HDBiCMOS Buffer } & \multicolumn{2}{c|}{ modified Proposed HDBiCMOS Buffer } \\
\cline { 2 - 7 } & $\begin{array}{c}\text { Propagation } \\
\text { Delay(S) }\end{array}$ & $\begin{array}{c}\text { Power } \\
\text { Dissipation } \\
(\mathbf{W})\end{array}$ & $\begin{array}{c}\text { PDP } \\
\text { (WS) }\end{array}$ & $\begin{array}{c}\text { Propagation } \\
\text { Delay(S) }\end{array}$ & $\begin{array}{c}\text { Power } \\
\text { Dissipation } \\
(\mathbf{W})\end{array}$ & $\begin{array}{c}\text { PDP } \\
\text { (WS) }\end{array}$ \\
\hline 20 & $1.91 \times 10^{-8}$ & $2.06 \times 10^{-4}$ & $3.95 \times 10^{-12}$ & $1.10 \times 10^{-8}$ & $3.68 \times 10^{-4}$ & $4.08 \times 10^{-12}$ \\
\hline 40 & $3.30 \times 10^{-8}$ & $2.20 \times 10^{-4}$ & $7.27 \times 10^{-12}$ & $2.07 \times 10^{-8}$ & $3.82 \times 10^{-4}$ & $7.91 \times 10^{-12}$ \\
\hline 60 & $4.90 \times 10^{-8}$ & $2.33 \times 10^{-4}$ & $1.14 \times 10^{-11}$ & $3.07 \times 10^{-8}$ & $3.95 \times 10^{-4}$ & $1.21 \times 10^{-11}$ \\
\hline 80 & $6.51 \times 10^{-8}$ & $2.48 \times 10^{-4}$ & $1.61 \times 10^{-11}$ & $4.08 \times 10^{-8}$ & $4.08 \times 10^{-4}$ & $1.66 \times 10^{-11}$ \\
\hline 100 & $8.11 \times 10^{-8}$ & $2.60 \times 10^{-4}$ & $2.11 \times 10^{-11}$ & $5.09 \times 10^{-8}$ & $4.22 \times 10^{-4}$ & $2.15 \times 10^{-11}$ \\
\hline 120 & $9.71 \times 10^{-8}$ & $2.74 \times 10^{-4}$ & $2.66 \times 10^{-11}$ & $6.09 \times 10^{-8}$ & $4.34 \times 10^{-4}$ & $2.65 \times 10^{-11}$ \\
\hline 140 & $1.13 \times 10^{-7}$ & $2.88 \times 10^{-4}$ & $3.26 \times 10^{-11}$ & $7.10 \times 10^{-8}$ & $4.48 \times 10^{-4}$ & $3.18 \times 10^{-11}$ \\
\hline 160 & $1.29 \times 10^{-7}$ & $3.02 \times 10^{-4}$ & $3.90 \times 10^{-11}$ & $8.11 \times 10^{-8}$ & $4.62 \times 10^{-4}$ & $3.74 \times 10^{-11}$ \\
\hline 180 & $1.45 \times 10^{-7}$ & $3.15 \times 10^{-4}$ & $4.58 \times 10^{-11}$ & $9.12 \times 10^{-8}$ & $4.74 \times 10^{-4}$ & $4.32 \times 10^{-11}$ \\
\hline 200 & $1.61 \times 10^{-7}$ & $3.28 \times 10^{-4}$ & $5.30 \times 10^{-11}$ & $1.01 \times 10^{-7}$ & $4.87 \times 10^{-4}$ & $4.93 \times 10^{-11}$ \\
\hline
\end{tabular}

\section{Conclusion}

Decreasing the delay value beside power consumption are among the main challenges in designing circuits. On the other hand, increasing the load capacitor value results in significant increase in delay and power consumption value. Therefore designing a buffer and placing it in circuit's output is of important factors since they are substantial in increasing the diving capability. The buffers presented in this paper are BiCMOS which operate properly in voltage range of $0.8 \mathrm{v}$ to $1.5 \mathrm{v}$. Such buffer enables charging and discharging capacitors with a low delay value. According to the simulation results with HSPICE simulator, the proposed circuits respond better with capacitor range between $0.5 \mathrm{pf}$ to $200 \mathrm{pf}$. Moreover, the delay value could also be decreased more by modified circuit.

The proposed circuit enjoys less delay value and better performance in comparison to the other similar circuits. It also improves the delay value about $88 \%$ compared to CMOS circuits for large capacitor value. However it is worth mentioning that, with increasing the capacitor value up to 200 pf and more, CMOS buffer circuits will have the lower frequency (about 25\%) than BiCMOS. In other words BiCMOS circuits operating frequency range is higher than the CMOS circuits.

\section{References}

[1] D. Kim, Y. Lee, J. Cai, I. Lauer, L. Chang, S. J. Koester, et al., "Low power circuit design based on heterojunction tunneling transistors (HETTs)," in Proceedings of the 14th ACM/IEEE international symposium on Low power electronics and design, 2009, pp. 219-224.

[2] G.Rajeshwari, Anjo.C.A, N.Arun Kumar, "Design of High Speed Array Multiplier using BiCMOS Logic for Driving Large Load", IJCA Proceedings on National Conference on VLSI and Embedded Systems, 2013.

[3] K.-S. Yeo, H.-K. Lee, and M.-A. Do, "A high-speed twin-capacitor BiNMOS (TC-BiNMOS) logic circuit for single battery operation," Circuits and Systems I: Fundamental Theory and Applications, IEEE Transactions on, vol. 48, pp. 399-405, 2001.

[4] H. Etemadi, M. F. Dabiri, P. Keshavarzian, and T. Panahi, "Design of CNTFET-Based invertor Inspired BiCMOS Technology." 
[5] P. Heydari and R. Mohavavelu, "Design of ultra-high-speed CMOS CML buffers and latches," in Circuits and Systems, 2003. ISCAS'03. Proceedings of the 2003 International Symposium on, 2003, pp. II-208-II211 vol. 2.

[6] S. H. Embabi, A. Bellaouar, and K. Islam, "A bootstrapped bipolar CMOS (B 2 CMOS) Gate for lowvoltage applications," Solid-State Circuits, IEEE Journal of, vol. 30, pp. 47-53, 1995.

[7] P. Heydari, "Design and analysis of low-voltage current-mode logic buffers," in Quality Electronic Design, 2003. Proceedings. Fourth International Symposium on, 2003, pp. 293-298.

[8] N. D. A. GHORBAN, K. Navi, and O. Hashemipour, "High Speed Full Swing Current Mode Bicmos Logical Operators," International Journal of Engineering, 2007.

[9] R. Y. Chik and C. A. T. Salama, "Design of a 1.5 V full-swing bootstrapped BiCMOS logic circuit," Solid-State Circuits, IEEE Journal of, vol. 30, pp. 972-978, 1995.

[10] Shebaita, A., \& Ismail, Y. (2008). Multiple threshold voltage design scheme for CMOS tapered buffers. Circuits and Systems II: Express Briefs, IEEE Transactions on, 55(1), 21-25.

[11] Sharma, D., \& Mehra, R. (2011). Low Power, Delay Optimized Buffer Design using 70nm CMOS Technology. International Journal of Computer Applications, 22(3), 13-18.

[12] Saxena, A., \& Kaushik, P. Design of CMOS Tapered Buffer for High Speed and Low Power Applications using $65 \mathrm{~nm}$ Technology.

\section{Authors' Profiles}

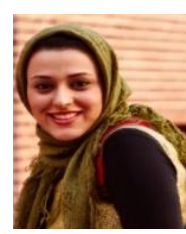

Maede Kaviani received the B.Sc degree in computer hardware and the M.Sc. Degree in computer architecture from Islamic Azad University (IAU), Najaf abad branch, Esfahan, Iran, in 2010 and 2015 respectively. Her interests are in the VLSI, circuit design and impact of technology on Computer Architecture

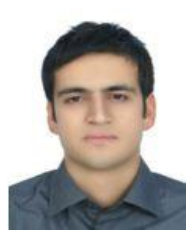

Hojjat Sharifi received his B.Sc degree in computer hardware and the M.Sc. Degree in computer architecture from Shahid Bahonar University of Kerman, Kerman, Iran, in 2005 and 2008 respectively. His research interests include VLSI Design, performance modeling and photonic integrated circuits.

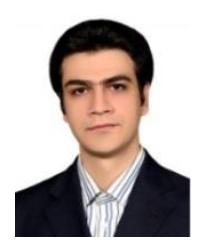

Mehdi Dolatshahi was born in Isfahan, Iran in 1980. He received the B.Sc. and M.Sc. degree in Electrical Engineering in 2003, 2006 respectively. He received the Ph.D. degree in Electrical Engineering in 2011 from Science and Research Branch, Islamic Azad University, Tehran, Iran. He has been with the Department of Electrical Engineering of Najafabad Branch, Islamic Azad University, since 2006 where he is currently an assistant professor. His research interests include VLSI and CMOS low-voltage, low power analog and mixed-signal integrated circuit design and optimization as well as CMOS optical communications circuit design. 
Keivan Navi received M.Sc. degree in electronics engineering from Sharif University of Technology, Tehran, Iran in 1990. He also received the Ph.D. degree in computer architecture from Paris XI University, Paris, France, in 1995. He is currently a Professor in Faculty of Electrical and Computer Engineering of Shahid Beheshti University. His research interests include Nanoelectronics with emphasis on CNFET, QCA and SET, Computer Arithmetic, Interconnection Network Design and Quantum Computing and cryptography.

How to cite this paper: Maede Kaviani, Hojjat Sharifi, Mahdi Dolatshahi, Keivan Navi,"Design of Low Voltage and High-Speed BiCMOS Buffer for Driving Large Load Capacitor", International Journal of Engineering and Manufacturing(IJEM), Vol.6, No.1, pp.1-9, 2016.DOI: 10.5815/ijem.2016.01.01 